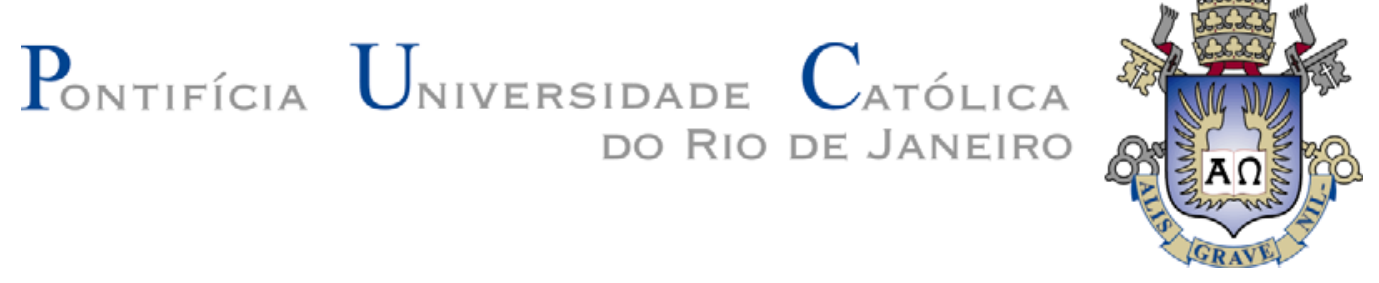

Valéria Debórtoli de Carvalho Queiroz

Entre o passado e o presente: a atuação do Assistente Social no campo da saúde mental

Dissertação de Mestrado

Dissertação apresentada ao Programa de PósGraduação em Serviço Social da PUC-Rio como requisito parcial para obtenção do título de Mestre em Serviço Social.

Orientador: Prof. ${ }^{a}$ Dr. ${ }^{\text {a }}$ Sueli Bulhões da Silva Co-Orientador: Prof. Dr. Eduardo Mourão Vasconcelos 


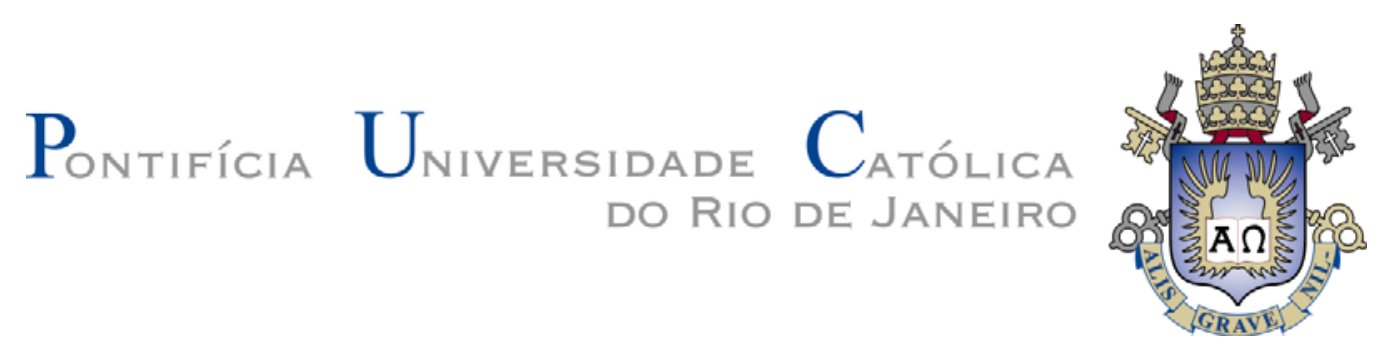

Valéria Debórtoli de Carvalho Queiroz

\section{Entre o passado e o presente: a atuação do Assistente Social no campo da saúde mental}

Dissertação apresentada ao Programa de Pós-Graduação em Serviço Social da PUC-Rio como requisito parcial para obtenção do título de Mestre em Serviço Social. Aprovada pela Comissão Examinadora abaixo assinada.

Prof. $^{a}$ Dr. ${ }^{a}$ Sueli Bulhões da Silva Orientadora Departamento de Serviço Social - PUC - Rio

Prof. Dr. Eduardo Mourão Vasconcelos Co-Orientador Departamento de Serviço Social - UFRJ

Prof. $^{a}$ Dr. ${ }^{a}$ Ludmila Fontenele Cavalcanti Departamento de Serviço Social - UFRJ

Prof. Dr. Erimaldo Matias Nicácio Departamento de Serviço Social - UFRJ

Prof. Nizar Messari Vice-Decano de Pós-Graduação do Centro de Ciências Sociais - PUC- Rio

Rio de Janeiro, 09 julho de 2009. 
Todos os direitos reservados. É proibida a reprodução total ou parcial do trabalho sem a autorização da universidade, da autora e do orientador.

Valéria Debórtoli de Carvalho Queiroz

Graduou-se em Serviço Social pela UFJF (Universidade Federal de Juiz de Fora) em 1996. Atuou no campo da saúde mental em Volta Redonda no período 1990-2000. Trabalhou como assistente social da Empresa de Saneamento do Município de Resende de 2000-2003.

Ficha Catalográfica

Queiroz, Valéria Debórtoli de Carvalho

"Entre o passado e o presente: a prática profissional do Assistente Social no campo da saúde mental", Valéria Debórtoli de Carvalho Queiroz: Orientadora Sueli Bulhões da Silva, Coorientador: Eduardo Mourão Vasconcelos- Rio de Janeiro: PUC, Departamento de Serviço Social.

$163 \mathrm{f}, 30 \mathrm{~cm}$.

1. Dissertação (Mestrado) - Pontifícia Universidade Católica do Rio de Janeiro, Departamento de Serviço Social,2009.

Inclui referências bibliográficas.

1. Serviço Social - Teses - 2 Saúde Mental. 3. Reforma Psiquiátrica - 4. Atuação Profissional. Silva, Sueli Bulhões da II Pontifícia Universidade Católica do Rio de Janeiro, Departamento de Serviço Social. III. Título 
Ao meu esposo e companheiro Sérgio que me incentivou para a concretização deste sonho e com quem compartilhei as minhas angústias, as alegrias e incertezas, sendo sempre o meu porto seguro. Ao meu filho Rafael que me motiva a sonhar e a almejar uma sociedade mais justa e igualitária. 


\section{Agradecimentos}

À minha orientadora Prof. ${ }^{a}$ Dr. ${ }^{a}$ Sueli Bulhões da Silva pela paciência, compreensão e sabedoria indispensáveis à realização deste trabalho.

Ao meu co-orientador Prof. Dr. Eduardo Mourão Vasconcelos pelo acolhimento, pelos conhecimentos no campo da saúde mental, pela disponibilidade e por acreditar em mim.

À Professora Dr. ${ }^{a}$ Maria Luiza Valente pelo carinho e pelos ensinamentos.

À PUC e à CAPES pelo apoio financeiro sem o qual este trabalho não poderia ser realizado.

Aos meus pais e irmãs que sempre estiveram dispostos a me ajudar, acolher e incentivar, apoiando-me incondicionalmente e infinitas vezes. Amo vocês!

Aos Assistentes Sociais da rede pública de atenção psicossocial de Juiz de Fora.

Aos meus colegas de mestrado de PUC-Rio pela troca de conhecimentos e pela amizade. 


\section{Resumo}

Queiroz, Valéria Debórtoli de Carvalho; Silva, Sueli Bulhões. Entre o passado e o presente: a prática profissional do Assistente Social no campo da saúde mental. Rio de Janeiro, 2009, 163p. Dissertação de Mestrado - Departamento de Serviço Social, Pontifícia Universidade Católica do Rio de Janeiro.

Esta dissertação diz respeito a atuação dos assistentes sociais no campo da saúde mental na cidade de juiz de Fora, MG - Brasil. Nosso objetivo principal foi conhecer como esta atuação tem se caracterizado após o processo da Reforma Psiquiátrica brasileira. Para conduzir o estudo, usamos uma abordagem qualitativa, apoiada em entrevistas semiestruturadas com assistentes sociais que atuam nos Centros de Atenção Psicossocial (CAPS) para adultos. Embora os resultados do estudo não possam generalizados, eles indicam que é necessário aprimorar as habilidades dos assistentes sociais que atuam no campo da saúde mental na cidade de Juiz de Fora.

\section{Palavras chave}

Serviço Social; saúde mental; reforma psiquiátrica. 


\section{Abstract}

Queiroz, Valéria Debórtoli de Carvalho; Silva, Sueli Bulhões (Advisor). Between the past to the present time: professional performance of the social worker in the mental health in Juiz de Fora. Rio de Janeiro, 2009, 163p. MSc. Dissertation Departamento de Serviço Social, Pontifícia Universidade Católica do Rio de Janeiro.

This dissertation deals with the performance of the social workers in the field of mental health in the city of Juiz de Fora, MG - Brazil. Our major goal was to understand how such performance has been characterized after the Brazilian Psychiatry Reform process. To conduct the study, we used a qualitative aproach supported by semi-structured interviews of social workers acting in the city Psychosocial Attention Center (CAPS) for adults. Although the study's findings can not be generalized they indicate that it is necessary to improve the skills of social workers acting in the field of mental health in the city of Juiz de Fora.

\section{Keywords}

Social work; mental health; psychiatric reform. 


\section{Sumário}

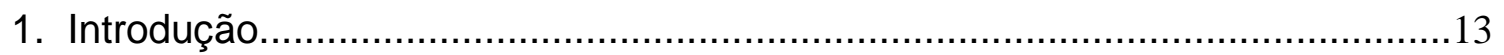

2. As formas históricas de abordagem da loucura até a virada do

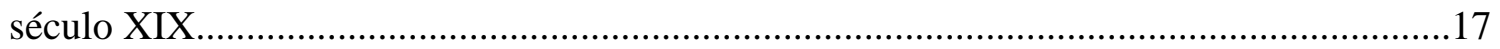

2.1. A grande internação no século XVII..............................................................18

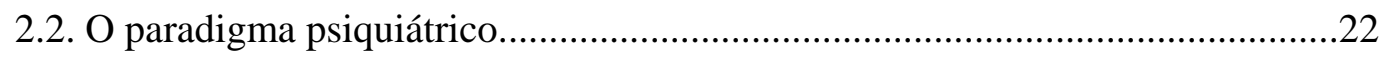

2.3. As características do manicômio como instituição total...................................25

2.4.As reformas psiquiátricas nos contextos europeu e americano...........................28

3. As políticas de saúde e de saúde mental no Brasil:

algumas reflexões.

3.1. O hospital D. Pedro II: o marco do nascimento da assistência

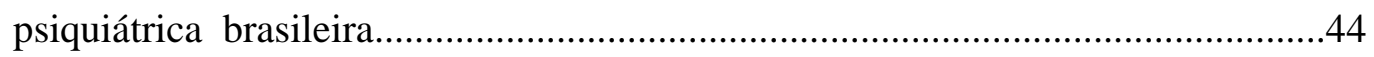

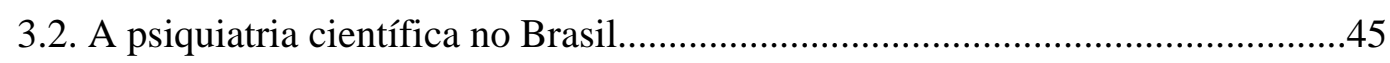

3.3. O movimento de reforma sanitária brasileira..................................................48

3.4. O processo de reforma psiquiátrica no Brasil.....................................................52

4. Serviço Social e Saúde Mental no século XX no Brasil....................................................66

4.1. A gênese e a profissionalização do Serviço Social no Brasil e sua inserção no campo da saúde mental........................................................................66

4.2. O debate atual do Serviço Social no campo da saúde mental.............................81

4.3. Os pontos de convergência entre o projeto ético-político do Serviço Social e os princípios da reforma psiquiátrica

5. O processo de reforma psiquiátrica em Juiz de Fora e a atuação

do assistente social. 
5.1 A política de Saúde Mental em Juiz de Fora.....................................................93

5.2 A descentralização e a política de saúde mental em

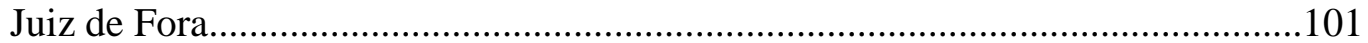

5.3. A pesquisa empírica e sua metodologia............................................................105

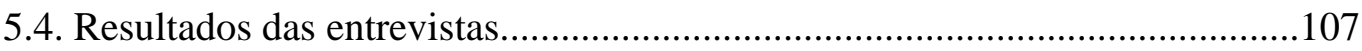

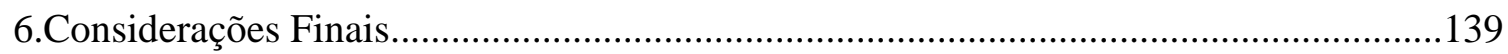

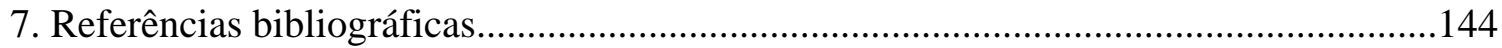

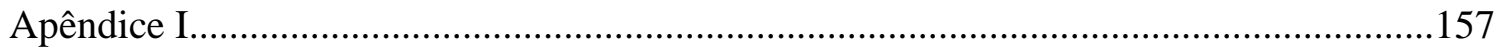

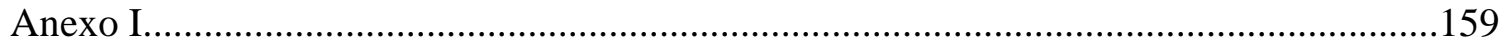

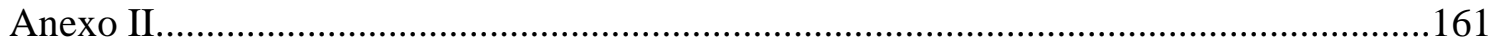




\section{Lista de Siglas}

HG - Hospital Geral

EUA - Estados Unidos da América

PDI - Psiquiatria Democrática Italiana

CAP's - Caixas de Aposentadorias e Pensões

IAP's - Institutos de Aposentadorias e Pensões

INPS - Instituto Nacional de Previdência Social

DINSAM - Divisão Nacional de Saúde Mental

REME - Movimento de Renovação Médica

MTSM - Movimento dos Trabalhadores da Saúde Mental

CONASP - Conselho Consultivo da Administração de Saúde Previdenciária

AIH - Autorização de Internação Hospitalar

NAPS - Núcleo de Atenção Psicossocial

USP - Universidade de São Paulo

PVC - Programa de Volta para Casa

ESF - Estratégia em Saúde da Família

PNASH - Programa Nacional do Sistema Hospitalar

MS - Ministério da Saúde

COI - Centros de Orientação Infantil

COJ - Centros de Orientação Juvenil

SMDM - Serviço Nacional de Doenças Mentais

INANPS - Instituto Nacional de Assistência Médica e Previdência Social

OMS - Organização Mundial de Saúde

OPS - Organização Pan-Americana da Saúde

PUC - Pontifícia Universidade Católica

CAPS - Centro de Atenção Psicossocial

CAPS’ad - Centro de Atenção Psicossocial Álcool e Drogas

CAPS’i - Centro de Atenção Psicossocial Infantil

CLI-JF - Comissão Local Interestadual de Juiz de Fora

AIS - Ações Integradas de Saúde

GT - Grupo de Trabalho 
CESM - Comissão Executiva de Saúde Mental

SES - Secretaria Estadual de Saúde

DRS - Diretoria Regional de Saúde

SUP - Serviço de Urgência Psiquiátrica

PSM - Pronto Socorro Municipal

FHEMIG - Fundação Hospitalar do Estado de Minas Gerais

UFJF - Universidade Federal de Juiz de Fora

ISM - Instituto de Saúde Mental

SRT - Serviço de Residência Terapêutica

SUS - Sistema Único de Saúde

CONEP - Conselho Nacional de Ética em Pesquisa

PJ - Partido Jovem

PTB - Partido Trabalhista Brasileiro

CRRESAM - Centros Regionais de Referência em Saúde Mental

UBS - Unidade Básica de Saúde

HP - Hospital Psiquiátrico

HPS - Hospital de Pronto Socorro 


\section{Lista de Tabelas}

Tabela 01: da Rede CAPS, cobertura PSF e ACS nas grandes cidades (mais 500.000 habitantes)

Tabela 02: Cobertura de CAPS por Unidade Federativa 62

Tabela 03: Concentração de Leitos psiquiátricos e leitos/1000 hab.UF. .63

Tabela 04: Leitos psiquiátricos SUS por ano (2002- out - 2008). .64

Tabela 05: STR em funcionamento (outubro/2008). .65 